\title{
Intervenções via Internet para redução do consumo de álcool entre universitários: uma revisão sistemática
}

\author{
André Bedendo, ${ }^{1}$ André Luiz Monezi Andrade ${ }^{2}$ e Ana Regina Noto ${ }^{1}$
}

Como citar Bedendo A, Andrade ALM, Noto AR. Intervenções via Internet para redução do consumo de álcool entre universitários: uma revisão sistemática. Rev Panam Salud Publica. 2018;42:e54. https://doi.org/ 10.26633/RPSP.2018.54

RESUMO Objetivo. Identificar as principais modalidades de intervenções via Internet para limitar o uso de álcool por estudantes universitários e descrever os efeitos dessas intervenções sobre o consumo e as consequências do uso de álcool.

Método. Esta é uma revisão sistemática com artigos disponíveis nas bases PubMed, PsycINFO e SciELO. Foram utilizados os termos de busca alcohol drinking AND Internet, sem restrição de data ou idioma. Foram critérios de inclusão: ser estudo randomizado controlado com amostra de estudantes universitários, enfocar intervenções via Internet e avaliar os efeitos dessas intervenções sobre o consumo ou as consequências do uso de álcool.

Resultados. Foram analisados 36 artigos e identificadas duas modalidades principais de intervenção via Internet: feedbacks normativos personalizados (FNP, $n=28$ ) e intervenções multicomponentes $(n=8)$. Doze estudos com FNP observaram redução no uso de álcool 3 meses após a intervenção. Intervenções multicomponentes reduziram o consumo e as consequências do uso. A intervenção mais avaliada foi o site AlcoholEdu, que reduziu o uso de álcool e as consequências do uso 6 meses após a intervenção.

Conclusões. FNP e o site AlcoholEdu foram as intervenções mais avaliadas entre os estudos selecionados e se mostraram eficazes na redução do consumo de álcool entre estudantes universitários. Essas estratégias são uma alternativa para ampliar o acesso de universitários a intervenções para limitar o uso de álcool.

Palavras-chave Consumo de bebidas alcoólicas; consumo de álcool na faculdade; Internet; universidades; revisão.

A prevalência de consumo de bebidas alcoólicas é alta entre universitários, sendo que, no Brasil, aproximadamente

1 Universidade Federal de São Paulo (UNIFESP), Núcleo de Estudos e Pesquisa em Saúde e Uso de Substâncias (NEPSIS), Departamento de Psicobiologia, São Paulo (SP), Brasil. Correspondência: Ana Regina Noto, arnpsicobio@gmail.com

2 Pontifícia Universidade Católica de Campinas (PUC-Campinas), Centro de Ciências da Vida Campus II, Campinas (SP), Brasil.
$70 \%$ dos estudantes relataram consumo de álcool no último ano e $60 \%$ no último mês (1). O uso de álcool por universitários está associado a problemas pessoais (prejuízo acadêmico, comportamento sexual de risco, suicídio, dirigir alcoolizado, overdoses), problemas envolvendo terceiros (vandalismo, violência física e sexual) e institucionais (custos legais, danos ao patrimônio, evasão estudantil) (2). Entretanto, poucos estudantes recebem tratamento ou intervenção precoces relacionados ao uso de álcool (3). No Brasil, poucas instituições possuem estratégias para redução dos problemas associados ao uso de álcool entre universitários (1), limitando a disponibilidade de acesso entre os estudantes.

Nos últimos anos, ampliou-se o número de pessoas com acesso regular à Internet. Assim, a Internet tem sido cada vez mais utilizada como meio para oferta de 
intervenções em saúde, inclusive relacionadas ao uso de álcool. As intervenções via Internet oferecem vantagens em relação aos modelos tradicionais (presenciais), inclusive boa relação custo-efetividade e acesso de grande número de pessoas simultaneamente $(4,5)$. Vale notar que, no Brasil, a maioria dos usuários da Internet tem idade entre 16 e 34 anos (6).

Revisões sistemáticas e metanálises realizadas anteriormente indicaram efeitos positivos de intervenções via Internet na redução do consumo de álcool e das consequências do uso dessa substância entre universitários (7-9). Contudo, esses estudos consideraram amostras mistas de intervenções via computador (em ambiente controlado/laboratório de pesquisa) e via Internet (possibilidade de acesso por computadores pessoais com livre acesso à Internet) (8). Isso limita o conhecimento específico sobre os efeitos de cada uma dessas modalidades, uma vez que os cenários de aplicação são bastante diferentes, podendo também gerar efeitos diferentes. Além disso, esses estudos apresentam limitações importantes, como considerar exclusivamente grupos restritos - por exemplo, estudantes no $1^{\circ}$ ano da universidade (9) -, inclusão de poucos artigos na revisão ou ausência de artigos em outras línguas que não o inglês, limitando a generalização dos resultados a outra culturas $(7,8)$.

Dessa forma, os objetivos deste estudo foram 1) identificar as principais modalidades de intervenções via Internet descritas na literatura para inibir o uso de álcool entre estudantes universitários e 2) descrever os efeitos dessas intervenções sobre o consumo e as consequências do uso de álcool.

\section{MATERIAIS E MÉTODOS}

A presente revisão sistemática da literatura foi realizada em fevereiro de 2016 a partir de busca na bases de dado PubMed, PsycINFO e SciELO. Os termos de busca utilizados foram alcohol drinking AND Internet, sem restrição de data ou idioma. A busca considerou a presença dos descritores em qualquer campo da referência (título, resumo ou texto completo), conforme detalhado a seguir: PubMed: ("alcohol drinking" [MeSH Terms] OR ("alcohol"[All Fields] AND "drinking"[All Fields]) OR "alcohol drinking"[All Fields]) AND
("Internet"[MeSH Terms] OR "Internet" [All Fields]); PsycINFO: Any Field: alcohol drinking AND Any Field: Internet; e SciELO: alcohol drinking AND Internet no campo All Fields. Os resultados das buscas foram organizados para triagem no software EndNote, sendo excluídos os artigos duplicados.

A busca nas bases de dados detectou 738 artigos (figura 1). A triagem desses artigos foi realizada inicialmente por título, seguida pela leitura dos resumos e dos textos completos, sendo incluídos na síntese qualitativa dos resultados 36 manuscritos que atendiam aos critérios de inclusão/exclusão. O conteúdo dos artigos foi avaliado por dois autores que atuaram como juízes de maneira independente. Os estudos foram catalogados em uma planilha contendo as informações de interesse para esta revisão. Casos de discordância entre os juízes foram discutidos em reuniões a fim de atingir concordância.

Foram coletados dados sobre autores, ano da publicação, tamanho de amostra, tempo de seguimento, instrumentos utilizados, uso de incentivos, tipos de intervenção e principais resultados dos estudos. A identificação e a apresentação dos resultados estão de acordo com os Preferred Reporting Items for Systematic Reviews and Meta-Analyses (PRISMA).

\section{Critérios de inclusão e exclusão}

Foram incluídos somente estudos a) randomizados, b) com amostra de estudantes universitários, c) avaliando efeitos de intervenções via Internet e d) com foco no consumo de álcool ou nas consequências associadas ao uso de álcool. Foram consideradas como consequências do uso de álcool quaisquer prejuízos associados ao uso de álcool relatados nos artigos.

Foram excluídas revisões da literatura e metanálises, protocolos de estudos e trabalhos de intervenção cuja amostra incluísse outros grupos etários (estudantes de ensino médio e população geral adulta). Além disso, também foram excluídos estudos que avaliaram intervenções sobre álcool em conjunto com outras drogas ou comportamentos em saúde (por exemplo, nutrição e exercício físico), ou estudos com indivíduos que possuíssem alguma comorbidade. A escolha de tais critérios visou à obtenção de relativa homogeneidade entre os estudos em relação a metodologia, características da população alvo e substância alvo da intervenção. Este foco favorece a identificação do contexto no qual as intervenções incluídas nesta revisão podem atingir os resultados esperados.

FIGURA 1. Fluxograma de triagem e seleção de artigos sobre intervenções via Internet para reduzir o consumo de álcool entre universitários

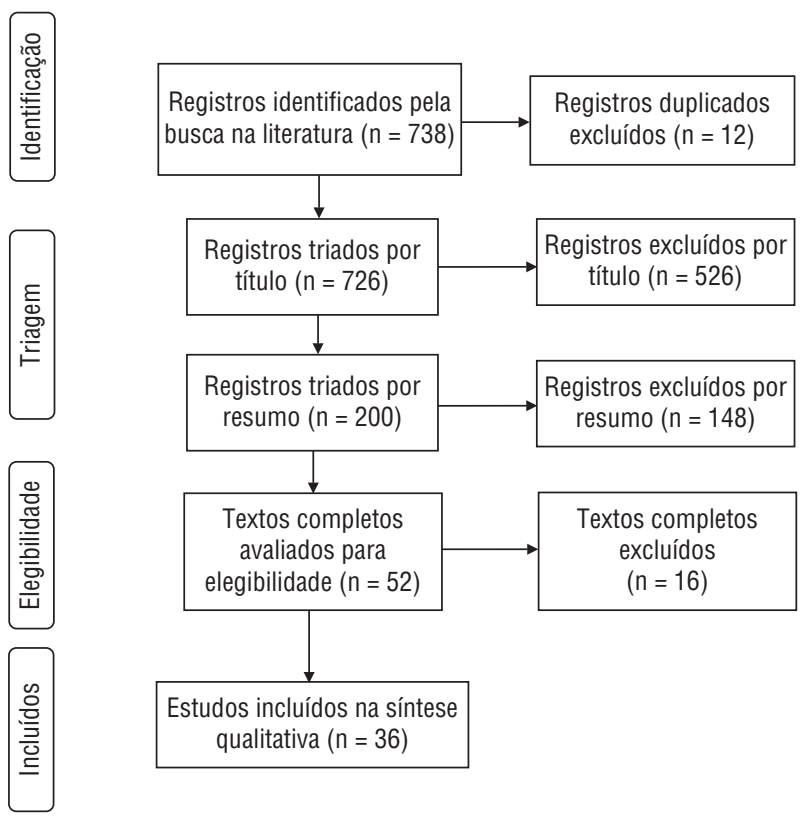




\section{RESULTADOS}

Como mostra a tabela 1, a maioria dos estudos foi realizada com amostras de universitários dos Estados Unidos ( $\mathrm{n}=21,58,3 \%$ ). Não foram encontrados estudos brasileiros ou de outros países da América do Latina, nem estudos em outro idioma além do inglês. A maioria dos estudos ( $n=26,72,2 \%$ ) apresentou tamanho de amostra grande (maior que 300), variando entre 67 (10) e 5074 estudantes $(11,12)$. O tempo de seguimento variou de 1 semana (13-15) a 24 meses (16), com a maioria dos estudos apresentando tempo inferior a 2 meses $(\mathrm{n}=15$, $41,7 \%$ ). Somente um estudo apresentou seguimento maior do que 12 meses. Em relação aos instrumentos utilizados, o Alcohol Use Disorders Identification Test (AUDIT) foi o instrumento utilizado com maior frequência ( $\mathrm{n}=14,38,9 \%)$, seguido pelo Daily Drinking Questionnaire (DDQ) ( $\mathrm{n}=10,27,8 \%$ ) e pelo Rutgers Alcohol Problem Index (RAPI) ( $\mathrm{n}=8,22,2 \%)$.

A tabela 2 descreve os principais resultados dos estudos avaliados bem como as principais modalidades de intervenção avaliadas. As intervenções foram classificadas em três modalidades principais: feedbacks normativos personalizados (FNP), uma intervenção caracterizada pela apresentação de feedback após a avaliação do perfil de uso de álcool do indivíduo por meio de questionários; intervenções multicomponentes, um conjunto mais amplo de conteúdos relacionados ao consumo de álcool e que variam entre cada intervenção; e outras intervenções, que incluiu aplicativos de celular, feedback informativo sem conteúdos normativos (ou seja, relativos ao consumo de álcool da população a que o indivíduo pertence) e intervenção baseada em vídeos. Dos 36 artigos incluídos, 28 (77,8\%) avaliaram intervenções por FNP. Dois estudos foram categorizados simultaneamente nas modalidades FNP e multicomponentes $(17,18)$ e um estudo foi categorizado simultaneamente em FNP e outras intervenções (19).

\section{Efeitos do feedback normativo personalizado}

O FNP, no qual um feedback é apresentado após avaliação do perfil de uso de álcool do indivíduo por meio de questionários, demanda pouco tempo ( $5 \mathrm{a}$ 20 minutos). O feedback inclui informações individualizadas sobre o consumo de álcool de cada participante associadas a informações normativas, ou seja, informações sobre o consumo de álcool da população à qual o indivíduo pertence. Entre os 28 estudos que utilizaram o FNP, $21(75,0 \%)$ descreveram o efeito da intervenção sobre o consumo de álcool. Desses, seis estudos $(21,4 \%)$ também descreveram os efeitos sobre as consequências negativas do uso de álcool. Por sua vez, sete avaliações do FNP (25,0\%) não observaram efeitos da intervenção. As variáveis utilizadas como desfecho variaram significativamente entre os estudos (tabela 2).

Dos 21 estudos que observaram redução somente sobre o consumo de álcool, $12(57,2 \%)$ relataram efeitos até 3 meses $(10,14,17,19-27)$, cinco $(23,8 \%)$ relataram efeitos de 4 a 6 meses (28-32) e quatro $(19,0 \%)$ relataram efeitos a partir dos 6 meses $(16,18,33,34)$. Os efeitos do FNP sobre o consumo de álcool foram observados independentemente do formato do feedback, ou seja, breve (com menos conteúdo, por exemplo, três páginas de feedback) ou longo (versão mais extensa e com mais conteúdo, por exemplo, seis páginas de feedback) $(25,28)$, e da frequência de aplicação (uma única vez ou com várias reapresentações) (20). Quatro $(19,0 \%)$ estudos também indicaram que os efeitos na redução do consumo foram mediados pelas percepções do universitário acerca do consumo de álcool de seus pares $(10,16,18,26)$.

Entre os seis estudos que, além dos efeitos sobre o consumo, também demonstraram redução nas consequências associadas ao uso de álcool, dois $(33,3 \%)$ observaram efeito até 3 meses $(19,27)$, um $(16,7 \%)$ observou efeitos de 4 a 6 meses $(24)$ e três $(50,0 \%)$ estudos observaram efeitos até 6 meses após a intervenção $(16,18,33)$. Ao contrário dos efeitos sobre o uso de álcool, alguns estudos sugeriram que a disponibilização repetida do feedback promoveu efeitos de longo prazo sobre as consequências do uso $(16,33)$. Por fim, sete estudos (25,0\%) não observaram efeitos significativos do FNP sobre o consumo de álcool ou as consequências de seu uso $(13,35-40)$.

\section{Efeitos das intervenções multicomponentes}

Essa modalidade apresenta um conjunto mais amplo de conteúdos relacionados ao consumo de álcool e que variam entre cada intervenção. Essas intervenções tendem a ser mais longas do que as baseadas em FNP, com duração que varia de 80 minutos (41) até 3 horas $(11,12,42)$.

Entre os oito $(22,2 \%)$ estudos que avaliaram os efeitos de intervenções multicomponentes, quatro $(50,0 \%)$ enfocaram o site AlcoholEdu (11, 12, 17, 43), dois $(25,0 \%)$ enfocaram adaptações para Internet da intervenção Brief Alcohol Screening and Intervention for College Students BASICS $(15,18)$, um $(12,5 \%)$ enfocou o site CollegeAlc (42) e um (12,5\%) enfocou MyStudentBody.com: Alcohol (41). As intervenções CollegeAlc, AlcoholEdu e MyStudentBody.com: Alcohol promoveram redução do consumo e das consequências do uso de álcool. Contudo, esses efeitos variaram em relação ao tempo de follow-up e características dos participantes. Dois dos trabalhos sobre o AlcoholEdu observaram redução no consumo e consequências do uso de álcool na avaliação de 6 meses, mas não após 12 meses $(11,12)$; contudo, em outros dois estudos, tais efeitos foram observados somente 1 mês após a intervenção $(17,43)$. Os efeitos do CollegeAlc sobre consumo e consequências negativas associadas ao álcool foram observados após 3 meses e foram observados somente entre indivíduos que apresentavam consumo de bebidas anterior ao ingresso na universidade (42), enquanto a intervenção MyStudentBody.com: Alcohol reduziu o consumo e as consequências do uso de álcool, porém os efeitos foram observados somente após 3 meses e entre mulheres (41).

Entre os estudos que avaliaram versões adaptadas do BASICS, um observou redução somente no consumo de álcool, sendo que os efeitos tiveram duração de 12 meses (18). Em contrapartida, uma versão do BASICS para Internet que enfocou um período curto sem aulas (recesso de primavera na universidade) não reduziu o consumo ou as consequências do uso do álcool (15).

\section{Efeitos de outras modalidades}

Três estudos (8,3\%) avaliaram outras modalidades: aplicativos de celular (um on-line e um off-line, sendo os resultados desse último omitidos, já que o estudo não está alinhado com o escopo desta revisão) (44); feedback informativo sem conteúdo normativo - feedback de balança de decisão (FBD) (19); e intervenção em vídeo (45). 
TABELA 1. Dados sobre país, amostra, tempo de seguimento e instrumentos utilizados para avaliar o consumo e as consequências do uso de álcool em intervenções via Internet para reduzir o consumo de álcool entre universitários ${ }^{a}$

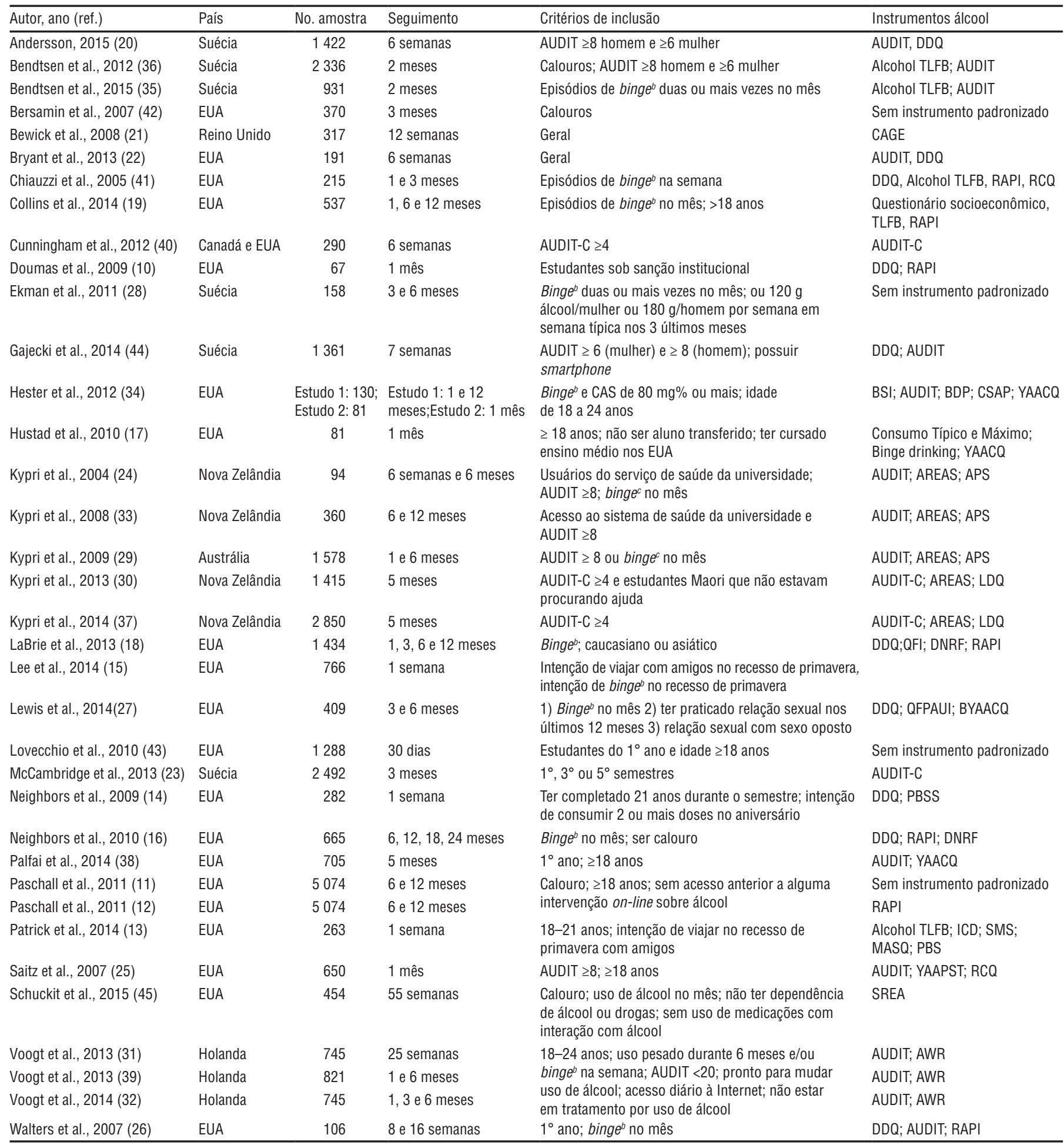

${ }^{a}$ APS: Alcohol Problems Scale; AREAS: Academic Role Expectations and Alcohol Scale; AUDIT: Alcohol Use Disorders Identification Test; AUDIT-C - Alcohol Use Disorders Identification Test Consumption Questions; AWR: Alcohol Weekly Recall; BDP: Brief Drinker's Profile; BSI: Brief Symptom Inventory; BYAACQ: Brief Young Adult Alcohol Consequences Questionnaire; CAS: concentração de álcool no sangue; CEAQ: Comprehensive Effects of Alcohol Questionnaire; CSAP: College Students Alcohol Problems; DDQ: Daily Drinking Questionnaire; DNRF: Drinking Norms Rating Form; ICD: Importance of Consequences of Drinking; LDQ: Leeds Dependence Questionnaire; MASQ: Motivations Against Sex Questionnaire; PBS: Protective Behavioral Strategies; PBSS: Protective Behavioral Strategies Scale; QFI: Quantity/Frequency Index; QFPAUI: Quantity/Frequency/Peak Alcohol Use Index; RAPI: Rutgers Alcohol Problem Index; RCQ: Readiness to Change Questionnaire; SMS: Sexual Motivations Scale; SREA: Self-Rating of the Effects of Alcohol; TLFB: Timeline Followback; YAACQ: Young Adult Alcohol Consequences Questionnaire; YAAPST: Young Adult Alcohol Problem Screening Test.

${ }^{\mathrm{b}}$ Binge $=5$ doses ou mais para homens e 4 doses ou mais para mulheres.

${ }^{c}$ Binge $=6$ doses ou mais para homens e 4 doses ou mais para mulheres. 

observados $^{\mathrm{a}}$

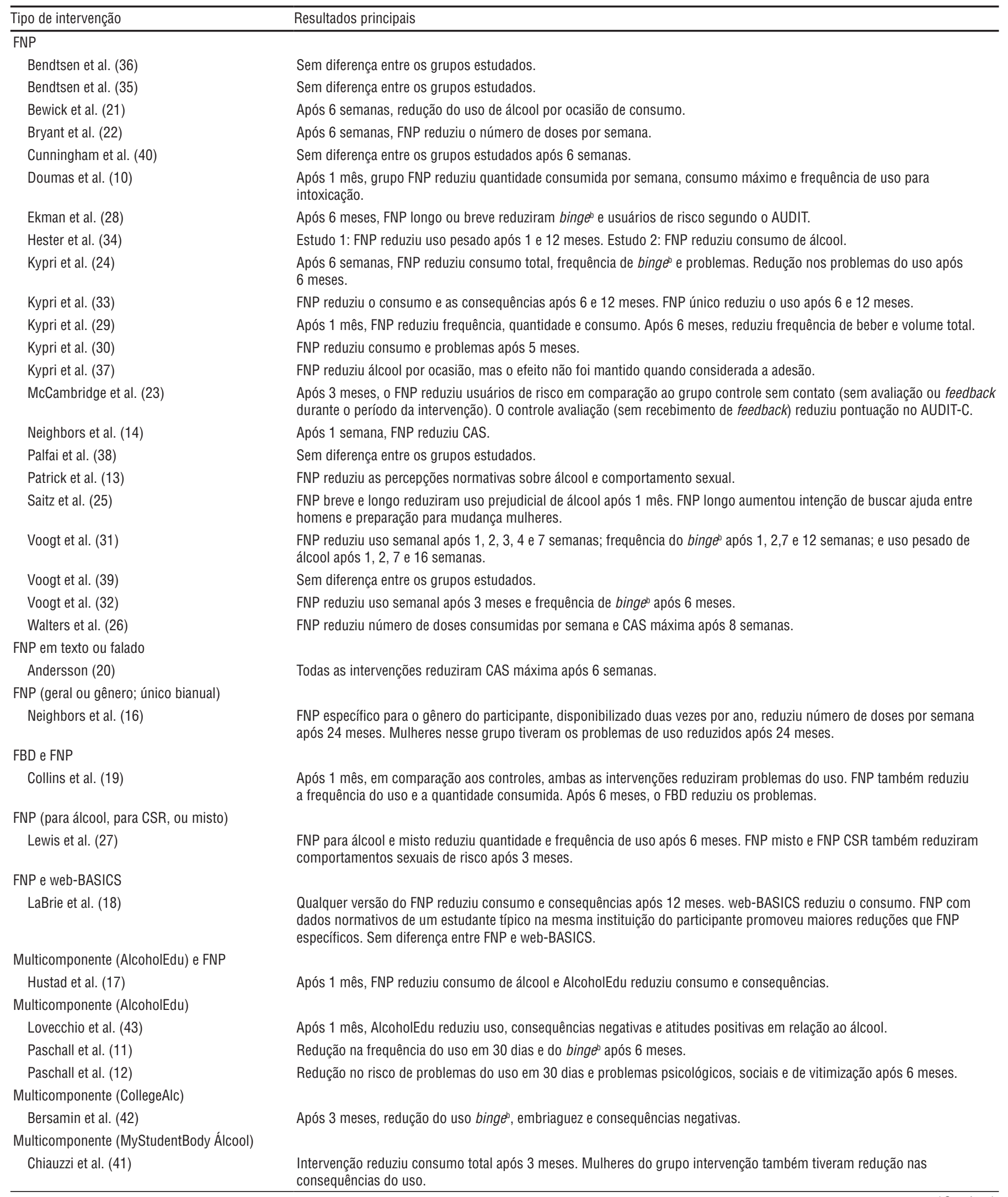


TABELA 2. (Continuação)

\begin{tabular}{|c|c|}
\hline Tipo de intervenção & Resultados principais \\
\hline \multicolumn{2}{|c|}{$\begin{array}{l}\text { Multicomponente (BASICS em versões padrão, } \\
\text { SB BASICS, presencial ou web) }\end{array}$} \\
\hline Lee et al. (15) & $\begin{array}{l}\text { Comparado ao controle, grupo SB BASICS presencial teve redução no CAS, número total e máximo de doses durante } 0 \\
\text { recesso de primavera após } 1 \text { semana. }\end{array}$ \\
\hline \multicolumn{2}{|l|}{ Vídeos } \\
\hline Schuckit et al. (45) & Comparados aos controles, participantes do grupo BRA reduziram consumo após 55 semanas. \\
\hline \multicolumn{2}{|l|}{ Aplicativo de celular } \\
\hline
\end{tabular}

O aplicativo de celular PartyPlanner permite planejamento ou simulação antecipados do consumo de álcool, além de fornecer em tempo real a concentração estimada de álcool no sangue. Contudo, não foram observados efeitos significativos sobre o consumo de álcool dos participantes que utilizaram o aplicativo (44). A intervenção por FBD apresentava informações individualizadas sobre as vantagens e desvantagens percebidas pelo estudante em relação ao consumo de álcool. Comparado ao grupo controle, o grupo com FBD teve redução no consumo e nas consequências do uso de álcool depois de 1 mês, sendo os efeitos sobre as consequências mantidos após 6 meses (19). Por fim, um trabalho comparou o efeito da apresentação de vídeos específicos para pessoas com baixa responsividade ao álcool - indivíduos que necessitam de maior concentração de álcool no sangue para atingir os efeitos esperados do uso ou sem ênfase neste aspecto. Pessoas com baixa responsividade que assistiram vídeos enfatizando essa característica apresentaram maior redução no consumo (45).

\section{Comparação entre diferentes modalidades de intervenções}

Três estudos (8,3\%) compararam diferentes modalidades de intervenção FNP versus multicomponentes $(17,18)$ e FNP versus outras modalidades (19). A comparação da intervenção multicomponente AlcoholEdu com FNP demonstrou que, comparadas a um grupo controle, ambas as intervenções promoveram redução no consumo de álcool 1 mês após a intervenção. AlcoholEdu também promoveu redução nas consequências associadas ao uso de álcool (17).

Também comparado a um grupo controle, o grupo com FNP teve redução no consumo e nas consequências do uso de álcool 12 meses após a intervenção, enquanto uma versão para Internet do BASICS reduziu somente o consumo de álcool nesse período. Contudo, não houve diferenças significativas entre os grupos FNP e web-BASICS (18). Em outro estudo, a comparação entre dois tipos de feedback (FNP e FBD) demonstrou que ambas as intervenções reduziram as consequências do uso 1 mês depois da intervenção, e que o FNP também reduziu a frequência e a quantidade do uso de álcool. Por sua vez, o FBD manteve seus efeitos sobre os problemas após 6 meses (19).

\section{Adesão e retenção dos participantes}

Um dos aspectos que se destacaram entre o conjunto de estudos, independentemente da modalidade de intervenção, foi a significativa perda de participantes, seja em termos de adesão (participantes que acessaram a avaliação inicial) ou retenção (participantes que acessaram as avaliações de seguimento). Apenas 23 estudos $(63,9 \%)$ apresentaram informações sobre as taxas de perda na adesão, com média de $73,5 \%$, variando de $45,3 \%$ $(17,25)$ a $97,1 \%$ (35). Por sua vez, as perdas de retenção se distribuíram da seguinte maneira: até $10 \%$ em oito estudos $(22,2 \%) ; 10,1$ a $20 \%$ em 10 estudos $(27,8 \%) ; 20,1$ a $30 \%$ em três estudos $(8,3 \%)$; 30,1 a $40 \%$ em seis estudos (16,7\%); 40,1 a $70 \%$ em quatro estudos (11,1\%); ou superiores a $71 \%$ em cinco estudos $(13,8 \%)$.

Como forma de reduzir as perdas, 31 trabalhos $(86,1 \%)$ utilizaram incentivos. Somente um $(2,8 \%)$ não utilizou incentivos $(35)$ e quatro $(11,1 \%)(10,22,23,28)$ não apresentaram informações sobre o uso ou não de incentivos. Vinte trabalhos $(55,6 \%)$ utilizaram incentivos em forma de dinheiro, que variaram em valor de 10 dólares $(11,12)$ até 135 dólares $(14,41)$ ou 100 euros $(31,32,39)$. Dez estudos $(27,8 \%)$ utilizaram incentivos em formato de brindes (tíquetes de alimentação, vale presente, entradas para eventos, iPhones e iPads). Um estudo utilizou como incentivo créditos para impressão de textos na universidade (21).

\section{Avaliação qualitativa dos artigos analisados}

Considerando aspectos como tamanho da amostra e clareza na apresentação da metodologia e dos resultados, os estudos apresentaram qualidade moderada, com descrição adequada dos métodos, cegamento quanto a alocação dos participantes, randomização e grupo controle. Vinte e cinco estudos $(69,4 \%)$ também apresentaram um tamanho de amostra grande (maior que 300), permitindo análises estatísticas minimamente adequadas. Entretanto, conforme apresentado anteriormente, as importantes perdas durante o follow-up limitam a compreensão sobre os efeitos das intervenções entre os indivíduos não aderentes.

Onze trabalhos (30,6\%) não apresentaram fluxograma da randomização e acompanhamento dos participantes (3, 14-23). Em particular, entre os estudos sobre FNP $(10,14,16-34)$, foi observada significativa heterogeneidade na descrição do tipo de intervenção, sendo utilizados termos diversos para denominar uma mesma modalidade, dificultando a identificação da mesma nos textos.

\section{DISCUSSÃO}

O presente estudo revisou a literatura científica acerca dos efeitos de intervenções via Internet sobre o consumo de álcool e as consequências negativas 
associadas a esse consumo entre estudantes universitários. Os dois tipos principais de intervenção identificados, FNP e multicomponentes, apresentaram efeitos positivos especialmente sobre a redução do consumo de álcool. Os efeitos do FNP foram mais robustos sobre o consumo de álcool até 3 meses após a intervenção. Entre as intervenções multicomponentes, o site AlcoholEdu foi a intervenção mais avaliada, apresentando efeitos positivos sobre consumo e consequências do uso de álcool que duraram por até 6 meses.

Apesar de os efeitos do FNP sobre o consumo de álcool terem sido observados eminentemente a curto prazo, efeitos de longo prazo foram observados a partir da disponibilização repetida do feedback $(16,33)$. Por sua vez, os efeitos sobre as consequências do uso são menos conclusivos, sendo ainda necessários estudos avaliando esse desfecho. Esses dados estão alinhados com resultados de uma metanálise que indicou efeitos maiores da intervenção sobre o uso de álcool do que sobre problemas relacionados ao uso (8). O tamanho de efeito nos estudos avaliando o FNP via Internet é modesto (16, 18), indicando uma moderada variação entre os grupos avaliados. Apesar dessa característica, devido à capacidade da intervenção de acessar grande número de pessoas de maneira eficiente e custoefetiva, seu impacto como política de saúde se mostra relevante (8). Contudo, destaca-se que esse impacto pode ser limitado por baixas taxas de adesão e retenção.

Alguns estudos indicaram que os efeitos do FNP sobre o consumo de álcool foram mediados por alterações nas percepções normativas dos participantes $(10,16,18,26)$. Entretanto, essas mudanças não parecem ser suficientes para modificar comportamentos de risco (13). Dessa forma, futuros estudos devem avaliar os mecanismos de mediação, ou seja, identificar como o FNP promove seus efeitos sobre comportamentos relacionados ao uso de álcool.

Houve grande variação nos aspectos metodológicos dos estudos avaliando o FNP, por exemplo em relação ao conteúdo dos feedbacks, tipo de grupo controle e critérios de inclusão. A utilização de diferentes componentes dos FNP pode estar associada ao fato de ainda não serem conhecidos os componentes mais efetivos do FNP (4). Em relação aos tipos de grupo controle, alguns estudos destacaram que somente uma avaliação sem apresentação de feedback também reduziu o consumo de álcool dos participantes $(23,34)$. Por fim, a utilização de diferentes critérios de inclusão (binge drinking definido como consumo de 5 ou mais doses para homens ou 4 ou mais para mulheres por semana; binge duas ou mais vezes por mês; AUDIT $\geq 4$; AUDIT $\geq 8$; entre outros) também favorece a heterogeneidade das amostras dos artigos, dificultando a comparação entre os estudos. Em conjunto, essas variações podem explicar o fato de alguns estudos observarem resultados positivos sobre os desfechos do uso de álcool, enquanto outros trabalhos não observam ou não replicam esses efeitos.

Entre as intervenções multicomponentes, a mais avaliada foi o site AlcoholEdu. A intervenção reduziu o consumo e as consequências do uso, sendo seus efeitos os mais duradouros nessa modalidade de intervenção. Comparado ao FNP, o AlcoholEdu promoveu redução no consumo e nas consequências associadas ao uso, enquanto o FNP reduziu somente o consumo de álcool (17). Esses dados corroboram uma recente iniciativa do National Institute on Alcohol Abuse and Alcoholism (NIAAA), o CollegeAIM, um guia informativo com dados para nortear a escolha de intervenções entre universitários. De acordo com o CollegeAIM, FNP e AlcoholEdu são estratégias individuais altamente efetivas para manejo do uso prejudicial de álcool entre universitários, ambas eficazes a curto prazo. Entretanto, destaca-se que os custos de implementação do AlcoholEdu são significativamente maiores do que os do FNP (46), um aspecto importante a ser considerado em uma possível aplicação dessas intervenções.

Poucos estudos avaliaram intervenções classificadas como outras modalidades (aplicativos de celular, vídeos ou FBD), limitando as conclusões sobre seus efeitos e sendo necessários mais estudos avaliando tais estratégicas. Entre essas alternativas, a versão que demonstrou resultados mais robustos foi o FBD, uma alternativa ao FNP para promover efeitos sobre as consequências do uso (19). Já o estudo avaliando a intervenção por vídeos (45) destaca a importância de fornecer conteúdo personalizado às características dos participantes para promover resultados positivos sobre o uso de álcool. Esse é um aspecto sugerido por outros autores como componente importante de intervenções via Internet (47). Por fim, o uso de uma intervenção via aplicativo de celular para planejamento de festas não é indicada por esta revisão como estratégia eficaz (44). Entretanto, isso não significa dizer que outros tipos de intervenção, como FNP ou intervenções multicomponentes, não serão efetivas se disponibilizadas nessa plataforma. Assim, ainda são necessários estudos avaliando o uso de outros aplicativos alternativos.

Diversos estudos desta revisão apresentaram significativa perda de participantes ao longo da intervenção. Como forma de contornar essa situação, a maioria dos estudos utilizou incentivos para reduzir as taxas de perda de participantes. Apesar de favorecer a adesão e a retenção de participantes, o uso de incentivos é pouco praticável durante a disseminação das intervenções (48) e pode comprometer a avaliação adequada da efetividade de intervenções via Internet (49). Além disso, um estudo recente destacou que indivíduos com maior consumo de álcool tendem a ser menos aderentes a intervenções via Internet, limitando a avaliação adequada dos efeitos dessas intervenções nesse grupo (50).

Os resultados deste estudo devem ser considerados em conjunto de suas limitações. Devido às características dos artigos encontrados nas buscas, os resultados não devem ser generalizados para o contexto brasileiro ou da América Latina, uma vez que não foram encontrados estudos avaliando o impacto das intervenções nessas regiões. Os artigos apresentados nesta revisão são provenientes de estudos publicados em periódicos científicos, não tendo sido considerada a literatura cinzenta, o que pode favorecer o viés de publicação. Apesar de a busca de artigos ter considerado palavras dos vocabulários controlados das bases de dados, não foram utilizados termos sinônimos, o que pode ter limitado a recuperação de alguns textos. Outra limitação se refere à heterogeneidade das intervenções em relação aos conteúdos apresentados e aos critérios de inclusão de cada trabalho. Por sua vez, entre os pontos fortes deste estudo estão a utilização das orientações PRISMA, a condução da revisão por dois autores, a utilização das principais bases de dados em saúde e a utilização de estudos randomizados e controlados, o que confere boa qualidade aos dados analisados. 


\section{CONCLUSÕES}

Foram identificadas três modalidades de intervenção via Internet para redução do uso ou das consequências do álcool entre estudantes universitários: FNP, intervenções multicomponentes e outras intervenções (aplicativos de celular, vídeos ou FBD). O FNP e as intervenções multicomponentes apresentaram efeitos sobre a redução do consumo de álcool, enquanto os efeitos sobre as consequências do uso foram menos robustos. Em relação às demais intervenções, as evidências de seus efeitos sobre o consumo de álcool e suas consequências ainda são limitadas.

A disponibilização de intervenções via Internet para estudantes pode favorecer

1. Andrade A, Duarte P, Oliveira L. I levantamento nacional sobre o uso de álcool, tabaco e outras drogas entre universitários das 27 capitais brasileiras. Brasília: SENAD; 2010.

2. White A, Hingson R. The burden of alcohol use: excessive alcohol consumption and related consequences among college students. Alcohol Res. 2013;35(2):201-18.

3. Wu LT, Pilowsky DJ, Schlenger WE, Hasin D. Alcohol use disorders and the use of treatment services among college-age young adults. Psychiatr Serv. 2007;58(2):192-200.

4. Riper H, van Straten A, Keuken M, Smit F, Schippers G, Cuijpers P. Curbing problem drinking with personalized-feedback interventions: a meta-analysis. Am J Prev Med. 2009;36(3):247-55.

5. White A, Kavanagh D, Stallman H, Klein B, Kay-Lambkin F, Proudfoot J, et al. Online alcohol interventions: a systematic review. J Med Internet Res. 2010;12(5):e62.

6. Comitê Gestor da Internet no Brasil. Pesquisa sobre o uso das tecnologias de informação e comunicação nos domicílios brasileiros: TIC domicílios 2015. São Paulo: 2016.

7. Bhochhibhoya A, Hayes L, Branscum P, Taylor L. The use of the Internet for prevention of binge drinking among the college population: a systematic review of evidence. Alcohol Alcohol. 2015;50(5): 526-35.

8. Dotson KB, Dunn ME, Bowers CA. Standalone personalized normative feedback for college student drinkers: a meta-analytic review, 2004 to 2014. PloS One. 2015;10(10): e0139518.

9. Scott-Sheldon LA, Carey KB, Elliott JC, Garey L, Carey MP. Efficacy of alcohol interventions for first-year college students: a meta-analytic review of randomized controlled trials. J Consult Clin Psychol. 2014;82(2):177-88.

10. Doumas DM, McKinley LL, Book P. Evaluation of two web-based alcohol interventions for mandated college students. J Subst Abuse Treat. 2009;36(1):65-74. o acesso de universitários em regiões onde há maior carência de informações sobre o uso de álcool ou entre estudantes que não acessam estratégias presenciais. Entretanto, a maioria das intervenções apresentou significativas perdas de participantes durante a avaliação ou uso de estratégias de recrutamento, o que pode ter enviesado os resultados. As estratégias via Internet devem ser consideradas em conjunto com outros tipos de intervenções que visem à redução do uso ou dos problemas associados ao consumo de álcool entre estudantes universitários.

Agradecimentos. À Fundação de Amparo à Pesquisa do Estado de São Paulo (FAPESP) pela bolsa de doutorado

\section{REFERÊNCIAS}

11. Paschall MJ, Antin T, Ringwalt CL, Saltz RF. Evaluation of an Internet-based alcohol misuse prevention course for college freshmen: findings of a randomized multicampus trial. Am J Prev Med. 2011;41(3): 300-8.

12. Paschall MJ, Antin T, Ringwalt CL, Saltz RF. Effects of AlcoholEdu for college on alcohol-related problems among freshmen: a randomized multicampus trial. J Stud Alcohol Drugs. 2011;72(4):642-50.

13. Patrick ME, Lee CM, Neighbors C. Webbased intervention to change perceived norms of college student alcohol use and sexual behavior on spring break. Addict Behav. 2014;39(3):600-6.

14. Neighbors C, Lee CM, Lewis MA, Fossos $\mathrm{N}$, Walter T. Internet-based personalized feedback to reduce 21st-birthday drinking: a randomized controlled trial of an eventspecific prevention intervention. J Consult Clin Psychol. 2009;77(1):51-63.

15. Lee CM, Neighbors C, Lewis MA, Kaysen D, Mittmann A, Geisner IM, et al. Randomized controlled trial of a Spring Break intervention to reduce high-risk drinking. J Consult Clin Psychol. 2014; 82(2):189-201.

16. Neighbors C, Lewis MA, Atkins DC, Jensen MM, Walter T, Fossos N, et al. Efficacy of web-based personalized normative feedback: a two-year randomized controlled trial. J Consult Clin Psychol. 2010;78(6):898-911.

17. Hustad JT, Barnett NP, Borsari B, Jackson KM. Web-based alcohol prevention for incoming college students: a randomized controlled trial. Addict Behav. 2010; 35(3):183-9.

18. Labrie JW, Lewis MA, Atkins DC, Neighbors C, Zheng C, Kenney SR, et al. RCT of web-based personalized normative feedback for college drinking prevention: are typical student norms good enough? J Consult Clin Psychol. 2013;81(6):1074-86.

19. Collins SE, Kirouac M, Lewis MA, Witkiewitz K, Carey KB. Randomized controlled trial of web-based decisional balance concedida ao primeiro autor. Os autores agradecem também à Coordenação de Aperfeiçoamento de Pessoal de Nível Superior (CAPES) e à Associação Fundo de Incentivo à Pesquisa (AFIP) pelo apoio técnico e financeiro, e ao Conselho Nacional de Pesquisa e Desenvolvimento (CNPq), pela bolsa de produtividade em pesquisa da última autora.

Conflitos de interesse. Nada declarado pelos autores.

Declaração. As opiniões expressas no manuscrito são de responsabilidade exclusiva dos autores e não refletem necessariamente a opinião ou políticas da RPSP/PAJPH ou da Organização Pan-Americana da Saúde (OPAS).

feedback and personalized normative feedback for college drinkers. J Stud Alcohol Drugs. 2014;75(6):982-92.

20. Andersson C. Comparison of WEB and interactive voice response (IVR) methods for delivering brief alcohol interventions to hazardous-drinking university students: a randomized controlled trial. Eur Addict Res. 2015;21(5):240-52.

21. Bewick BM, Trusler K, Mulhern B, Barkham M, Hill AJ. The feasibility and effectiveness of a web-based personalised feedback and social norms alcohol intervention in UK university students: a randomised control trial. Addict Behav. 2008; 33(9):1192-8.

22. Bryant ZE, Henslee AM, Correia CJ. Testing the effects of e-mailed personalized feedback on risky alcohol use among college students. Addict Behav. 2013;38(10):2563-7.

23. McCambridge J, Bendtsen M, Karlsson N, White IR, Nilsen P, Bendtsen P. Alcohol assessment and feedback by email for university students: main findings from a randomised controlled trial. Br J Psychiatry. 2013;203(5):334-40.

24. Kypri K, Saunders JB, Williams SM, McGee RO, Langley JD, Cashell-Smith ML, et al. Web-based screening and brief intervention for hazardous drinking: a double-blind randomized controlled trial. Addiction. 2004;99(11):1410-7.

25. Saitz R, Palfai TP, Freedner N, Winter MR Macdonald $\mathrm{A}, \mathrm{Lu} \mathrm{J}$, et al. Screening and brief intervention online for college students: the ihealth study. Alcohol Alcohol. 2007;42(1):28-36.

26. Walters ST, Vader AM, Harris TR. A controlled trial of web-based feedback for heavy drinking college students. Prev Sci. 2007;8(1):83-8.

27. Lewis MA, Patrick ME, Litt DM, Atkins DC, Kim T, Blayney JA, et al. Randomized controlled trial of a web-delivered personalized normative feedback intervention to reduce alcohol-related risky sexual behavior among college students. J Consult Clin Psychol. 2014;82(3):429-40. 
28. Ekman DS, Andersson A, Nilsen P, Stahlbrandt H, Johansson AL, Bendtsen P. Electronic screening and brief intervention for risky drinking in Swedish university students--a randomized controlled trial. Addict Behav. 2011;36(6):654-9.

29. Kypri K, Hallett J, Howat P, McManus A, Maycock B, Bowe S, et al. Randomized controlled trial of proactive web-based alcohol screening and brief intervention for university students. Arch Intern Med. 2009;169(16):1508-14.

30. Kypri K, McCambridge J, Vater T, Bowe SJ, Saunders JB, Cunningham JA, et al. Webbased alcohol intervention for Maori university students: double-blind, multi-site randomized controlled trial. Addiction. 2013;108(2):331-8.

31. Voogt CV, Kuntsche E, Kleinjan M, Poelen EA, Lemmers LA, Engels RC. Using ecological momentary assessment in testing the effectiveness of an alcohol intervention: a two-arm parallel group randomized controlled trial. PloS One. 2013;8(11):e78436.

32. Voogt C, Kuntsche E, Kleinjan M, Poelen E, Engels R. Using ecological momentary assessment to test the effectiveness of a web-based brief alcohol intervention over time among heavy-drinking students: randomized controlled trial. J Med Internet Res. 2014;16(1):e5.

33. Kypri K, Langley JD, Saunders JB, CashellSmith ML, Herbison P. Randomized controlled trial of web-based alcohol screening and brief intervention in primary care. Arch Intern Med. 2008;168(5):530-6.

34. Hester RK, Delaney HD, Campbell W. The college drinker's check-up: outcomes of two randomized clinical trials of a computer-delivered intervention. Psychol Addict Behav. 2012;26(1):1-12.

35. Bendtsen $P$, Bendtsen $M$, Karlsson N, White IR, McCambridge J. Online alcohol assessment and feedback for hazardous and harmful drinkers: findings from the AMADEUS-2 randomized controlled trial of routine practice in Swedish universities. J Med Internet Res. 2015;17(7):e170.
36. Bendtsen P, McCambridge J, Bendtsen M, Karlsson N, Nilsen P. Effectiveness of a proactive mail-based alcohol Internet intervention for university students: dismantling the assessment and feedback components in a randomized controlled trial. J Med Internet Res. 2012;14(5):e142.

37. Kypri K, Vater T, Bowe SJ, Saunders JB, Cunningham JA, Horton NJ, et al. Webbased alcohol screening and brief intervention for university students: a randomized trial. JAMA. 2014;311(12):1218-24.

38. Palfai TP, Winter M, Lu J, Rosenbloom D, Saitz R. Personalized feedback as a universal prevention approach for college drinking: a randomized trial of an e-mail linked universal web-based alcohol intervention. J Prim Prev. 2014;35(2):75-84.

39. Voogt CV, Poelen EA, Kleinjan M, Lemmers LA, Engels RC. The effectiveness of the 'what do you drink' web-based brief alcohol intervention in reducing heavy drinking among students: a two-arm parallel group randomized controlled trial. Alcohol Alcohol. 2013 ;48(3):312-21.

40. Cunningham JA, Hendershot CS, Murphy M, Neighbors C. Pragmatic randomized controlled trial of providing access to a brief personalized alcohol feedback intervention in university students. Addict Sci Clin Pract. 2012;7(1):21.

41. Chiauzzi E, Green TC, Lord S, Thum C, Goldstein M. My student body: a high-risk drinking prevention web site for college students. J Am Coll Health. 2005;53(6): 263-74.

42. Bersamin M, Paschall MJ, FearnowKenney M, Wyrick D. Effectiveness of a Web-based alcohol-misuse and harm-prevention course among high- and low-risk students. J Am Coll Health. 2007;55(4): 247-54.

43. Lovecchio CP, Wyatt TM, DeJong W. Reductions in drinking and alcohol-related harms reported by first-year college students taking an online alcohol education course: a randomized trial. J Health Commun. 2010;15(7):805-19.
44. Gajecki M, Berman AH, Sinadinovic K, Rosendahl I, Andersson C. Mobile phone brief intervention applications for risky alcohol use among university students: a randomized controlled study. Addict Sci Clin Pract. 2014;9:11.

45. Schuckit MA, Smith TL, Kalmijn J, Skidmore J, Clausen P, Shafir A, et al. The impact of focusing a program to prevent heavier drinking on a pre-existing phenotype, the low level of response to alcohol. Alcohol Clin Exp Res. 2015;39(2):308-16.

46. National Institute on Alcohol Abuse and Alcoholism. Planning Alcohol Interventions Using NIAAA's COLLEGE AIM Alcohol Intervention Matrix. 2015 Disponível em: https: / / www.collegedrinkingprevention. gov / CollegeAIM / Resources / NIAAA College_Matrix_Booklet.pdf Acessado em 21 de dezembro de 2017.

47. Cunningham JA, Murphy M, Hendershot CS. Treatment dismantling pilot study to identify the active ingredients in personalized feedback interventions for hazardous alcohol use: randomized controlled trial. Addict Sci Clin Pract. 2014;10:1

48. Geraghty AW, Torres LD, Leykin Y, PérezStable EJ, Muñoz RF. Understanding attrition from international Internet health interventions: a step towards global eHealth. Health Promot Int. 2013;28(3): 442-52.

49. Bedendo A, Noto AR. Studying an unreal world: incentives on Internet-based interventions for alcohol use. Addiction. 2016;111(2):373-4.

50. Radtke T, Ostergaard M, Cooke R, Scholz U. Web-based alcohol intervention: study of systematic attrition of heavy drinkers. J Med Internet Res. 2017;19(6):e217.

Manuscrito recebido em 11 de agosto de 2017. Aceito em versão revisada em 14 de dezembro de 2017. 
ABSTRACT Objective. To identify the main modalities of Internet-based interventions to limit alcohol use among university students, and to describe the effects of these interventions on alcohol use and related consequences.

Internet-based alcohol interventions for college students: systematic review

Keywords
Methods. A systematic review of PubMed, PsycINFO, and SciELO was performed using as search terms "alcohol drinking AND Internet," without date or language restrictions. Inclusion criteria were randomized controlled trial design, focus on college students and on Internet-based interventions, and evaluating the effects of the intervention on alcohol use or alcohol-related consequences.

Results. Thirty-six articles were analyzed. Two main intervention modalities were identified: personalized normative feedback (PNF, n=28) and multicomponent interventions $(n=8)$. Twelve studies with PNF reported reductions in alcohol use 3 months after the intervention. Multicomponent interventions reduced alcohol use and related consequences; most studies focused on the website AlcoholEdu, which reduced alcohol consumption and the consequences of alcohol use 6 months after the intervention.

Conclusions. FNP and the AlcoholEdu website, the most frequently evaluated interventions among the selected studies, were effective in reducing alcohol use in university students. These strategies are an alternative to increase the access of university students to interventions aimed at limiting alcohol use.

Alcohol drinking; alcohol drinking in college; Internet; universities; review.
RESUMEN

Intervenciones por Internet para reducir el consumo de alcohol de los estudiantes universitarios: revisión sistemática

Palabras clave
Objetivo. Determinar las principales modalidades de intervención por Internet para limitar el consumo de alcohol de los estudiantes universitarios, y describir los efectos de cada una en el consumo de alcohol y sus consecuencias.

Método. Esta es una revisión sistemática hecha con artículos disponibles en las bases PubMed, PsycINFO y SciELO. Se utilizaron como términos de búsqueda alcohol drinking AND Internet, sin restricción de fecha ni de idioma. Los criterios de inclusión fueron los siguientes: estudio aleatorizado controlado con una muestra de estudiantes universitarios, enfoque en intervenciones por Internet y evaluación de los efectos de esas intervenciones en el consumo de alcohol o sus consecuencias.

Resultados. El análisis de 36 artículos permitió encontrar dos modalidades principales de intervención por Internet, a saber, intervenciones basadas en retroalimentación normativa personalizada $(n=28)$ e intervenciones multicomponentes $(n=8)$. En doce estudios con retroalimentación normativa personalizada se observó una reducción del consumo de alcohol tres meses después de la intervención. Las intervenciones multicomponentes redujeron el consumo de alcohol y sus consecuencias. La intervención más evaluada fue el sitio AlcoholEdu, que disminuyó el consumo de alcohol y sus consecuencias seis meses después de realizarla.

Conclusiones. La retroalimentación normativa personalizada y el sitio AlcoholEdu fueron las intervenciones más evaluadas en los estudios seleccionados y demostraron ser eficaces para reducir el consumo de alcohol en los estudiantes universitarios. Esas estrategias ofrecen una alternativa para ampliar el acceso de los estudiantes a intervenciones destinadas a limitar el consumo de alcohol.

Consumo de bebidas alcohólicas; consumo de alcohol en la universidad; Internet; universidades; revisión. 